\title{
Comparative proteomics analysis of primary cutaneous amyloidosis
}

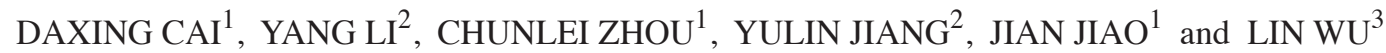 \\ ${ }^{1}$ Department of Dermatology, Qilu Hospital, Shandong University, Jinan, Shandong 250012; \\ ${ }^{2}$ Beijing Protein Innovation Co., Ltd., Beijing 101318; ${ }^{3}$ Key Laboratory of Genome Sciences and Information, \\ Beijing Institute of Genomics, Chinese Academy of Sciences, Beijing 100101, P.R. China
}

Received August 16, 2016; Accepted May 25, 2017

DOI: $10.3892 /$ etm.2017.4852

\begin{abstract}
Primary cutaneous amyloidosis (PCA) is a localized skin disorder that is characterized by the abnormal deposition of amyloid in the extracellular matrix (ECM) of the dermis. The pathogenesis of PCA is poorly understood. The objective of the present study was to survey proteome changes in PCA lesions in order to gain insight into the molecular basis and pathogenesis of PCA. Total protein from PCA lesions and normal skin tissue samples were extracted and analyzed using the isobaric tags for relative and absolute quantitation technique. The function of differentially expressed proteins in PCA were analyzed by gene ontology (GO), Kyoto Encyclopedia of Genes and Genomes (KEGG) and protein-protein interaction analysis. The proteins that were most upregulated in PCA lesions were further analyzed by immunohistochemistry. A total of 1,032 proteins were identified in PCA lesions and control skin samples, with 51 proteins differentially expressed in PCA lesions, of which 27 were upregulated. In PCA lesions, the upregulated proteins were primarily extracellulary located. In addition, GO analysis indicated that the upregulated proteins were significantly enriched in the biological processes of epidermal development, collagen fiber organization and response to wounding
\end{abstract}

Correspondence to: Dr Daxing Cai, Department of Dermatology, Qilu Hospital, Shandong University, 107 Wenhuaxi Road, Jinan, Shandong 250012, P.R. China

E-mail: caidaxing@medmail.com.cn

Dr Lin Wu, Key Laboratory of Genome Sciences and Information, Beijing Institute of Genomics, Chinese Academy of Sciences, 1-104 Beichenxi Road, Beijing 100101, P.R. China

E-mail:wul@big.ac.cn

Abbreviations: ECM, extracellular matrix; GO, gene ontology; IHC, immunohistochemistry; iTRAQ, isobaric tags for relative and absolute quantitation; LA, lichen amyloidosis; PCA, primary cutaneous amyloidosis; PPI, protein-protein interaction

Key words: primary cutaneous amyloidosis, iTRAQ, skin, amyloidosis (adjusted $\mathrm{P}<0.001)$. KEGG analysis indicated that the upregulated proteins were significantly enriched in the signaling pathways of cell communication, ECM receptor interaction and focal adhesion (adjusted $\mathrm{P}<0.001$ ). Furthermore, the upregulated proteins were enriched in the molecular function of calcium ion binding, and the calcium binding proteins calmodulin-like protein 5, S100 calcium-binding protein A7 (S100A7)/fatty-acid binding protein and S100A8/A9 exhibited the highest levels of upregulation in PCA. This analysis of differentially expressed proteins in PCA suggests that increased focal adhesion, differentiation and wound healing is associated with the pathogenesis of PCA.

\section{Introduction}

Amyloidosis is a heterogeneous group of diseases characterized by the deposition of amyloid in the ECM, with pathological consequences on the affected tissues or organs (1). Primary cutaneous amyloidosis (PCA) is a localized amyloidosis that affects only the skin, resulting in discolored bumps or nodules, and can be further divided into lichen amyloidosis (LA), macular amyloidosis and nodular amyloidosis (2). The course of the PCA is prolonged; patients often experience severe itchiness, seriously affecting their daily lives (3). PCA has a higher incidence in Asian populations (4). Pathogenic factors for PCA include prolonged friction, and genetic and environmental causes; however, the precise molecular mechanisms underlying the pathogenesis of PCA remain unclear (5).

Numerous previous studies investigating amyloidosis, including PCA, have focused on the identification and classification of the precursor proteins in amyloid deposits, since the pathological process and effects of treatment are associated with the type of amyloid precursor (6). To date, $>20$ types of amyloid precursors have been identified, and cytokeratins (CKs), serum amyloid $\mathrm{P}$, apolipoprotein E (ApoE), galectins and actins have been observed in PCA amyloid deposits (7-10). Previous studies on the genetic causes of PCA have identified mutations in several genes, including those encoding oncostatin $\mathrm{M}$ receptor and interleukin-31 receptor A $(11,12)$. A previous study noted a reduction in the expression of several interfollicular keratinocyte stem cell markers, and differences in the expression of proteins involved in apoptosis and nerve conduction, in PCA samples (13). However, to the best of our 
knowledge, a survey of proteome changes in PCA has not yet been reported.

Comparative proteomics has been successfully used to identify differentially expressed proteins in various disease conditions and provide insights into the molecular mechanisms underlying these diseases, in addition to diagnostic candidates. For example, Lu et al (14) compared the proteome of islets between a type 2 diabetes mouse model and nondiabetes control mice, and identified differential proteins related to insulin-secretory defects and mutiple novel proteins related to diabetes. Also using a comparative proteomics stretegy, Masui et al (15) identified 29 proteins differentially expressed in metastatic and primary renal cell carcinoma, among which profilin-1, 14-3-3 $\zeta$ and galectin-1 were demonstrated to be associated with poor prognosis. In the present study, a comparative proteomics study of PCA was performed, which identified numerous differentially expressed proteins, providing novel insights into the pathology of the disease.

\section{Materials and methods}

Tissue samples. Skin tissues from 10 patients diagnosed with LA (26-64 years old; 4 males and 6 females) and 10 healthy individuals (2-60 years old; 5 males and 5 females) were collected from January 2009 to December 2013 at Qilu Hospital (Shandong, China) for quantitative proteomics analysis. Additional skin samples from 29 patients diagnosed with LA (29-71 years old; 15 males and 14 females) and 17 healthy individuals (18-70 years old; 9 males and 8 females) were also collected from January 2009 to December 2013 at Qilu Hospital for immunohistochemistry (IHC) experiments. The diagnosis of LA was made by two physicians according to the diagnostic standards for PCA and histopathological examination (2). All PCA samples were also validated by crystal violet staining. For quantitative proteomics analysis, lesions with a size of $1 \times 2 \mathrm{~cm}$ were obtained surgically, immediately frozen in liquid nitrogen and then stored at $-80^{\circ} \mathrm{C}$ until required. For IHC analysis, skin tissue samples were fixed at room temperature with $4 \%$ formaldehyde for $24 \mathrm{~h}$ and formaldehyde (4\%)-acetic acid (5\%)-ethanol (45\%) for a further $3 \mathrm{~h}$, followed by stepwise dehydration with $80 \%$ ethanol for $2 \mathrm{~h}$, $90 \%$ ethanol for $3 \mathrm{~h}, 95 \%$ ethanol overnight, $100 \%$ ethanol twice $(20 \mathrm{~min} /$ treatment $)$ and xylene twice $(20 \mathrm{~min} /$ treatment $)$. The fixed and dehydrated tissues were immersed in paraffin wax twice (30 min/treatment) and then embedded into paraffin blocks until use. The present study was approved by the Ethics Committee of Qilu Hospital. All patients provided written informed consent for the inclusion of their skin samples in the present study.

Sample preparation for mass spectrometry analysis. Tissue samples from each group were combined and ground into powder in liquid nitrogen. The powders were washed once with cold trichloroacetic acid/acetone at $-20^{\circ} \mathrm{C}$ for $2 \mathrm{~h}$, twice with cold acetone at $-20^{\circ} \mathrm{C}$ for $30 \mathrm{~min}$ each, lyzed in lysis buffer ( $8 \mathrm{M}$ urea, $30 \mathrm{mM}$ hydroxyethyl piperazineethanesulfonic acid, $1 \mathrm{mM}$ phenylmethylsulfonyl fluoride, $2 \mathrm{mM}$ EDTA and $10 \mathrm{mM}$ dithiothreitol) and sonicated. The proteins in the supernatants were precfigipitated with acetone and resuspended in resuspension buffer [50\% triethylammonium bicarbonate (TEAB) and $0.1 \%$ SDS]. Total protein concentration was determined using the Bradford method (16). Proteins $(100 \mu \mathrm{g})$ were digested with $3.3 \mu \mathrm{g}$ of sequencing grade trypsin at $37^{\circ} \mathrm{C}$ for $24 \mathrm{~h}$ and with an additi onal $1 \mu \mathrm{g}$ for a further $12 \mathrm{~h}$. The digested proteins were frozen and lyophilized overnight in vacuum and resuspended in $61 \mu \mathrm{l}$ TEAB solution. The digestion efficiency was assessed with UltraFlex MALDI-TOF/TOF (Bruker Corporation, Ettlingen, Germany) to see whether there were a large number of peaks with signal-to-noise ratio $>10$ and $\geq 20$ peaks with intensities $>1,000$ within $\mathrm{m} / \mathrm{z}$ of $500-3,000$ Da.

Isobaric tags for relative and absolute quantitation (iTRAQ) labeling of peptides. Tryptic peptides from healthy and diseased groups were labeled with two different isobaric tags, using iTRAQ reagents 8-plex (SCIEX, Framingham, MA, USA) according to the manufacturer's protocol. Briefly, each iTRAQ reagent was dissolved in $70 \mu \mathrm{l}$ isopropanol, then added to each specimen prior to a $2 \mathrm{~h}$ incubation at room temperature. The labeled peptides were then vacuum-dried.

Liquid chromatography tandem-mass spectrometry (LC-MS/MS) analysis. All iTRAQ-labeled tryptic peptides from the two groups were combined for relative quantification analysis by LC-MS/MS using a Q Exactive ${ }^{\mathrm{TM}}$ Hybrid Quadrupole-Orbitrap Mass Spectrometer (Thermo Fisher Scientific, Inc., Waltham, MA, USA). The combined samples were first fractionated into 30-40 fractions by strong cation exchange chromatography (Luna SCX, 4.6x250 mm, Phenomenex, Torrance, CA, USA) as described previously (17). After desalting on a C18 column, the relative amount of peptides in each fraction was assessed by MALDI/TOF, and neighboring fractions with lower signals were pooled, yielding 10 fractions. Each combined fraction was subjected to MS in the standard MS/MS data-dependent acquisition mode. The MS spectra were surveyed from 350-2,000 Da and the method of collision-induced dissociation was chosen.

Data analysis for peptide identification and quantification. Protein identification and quantification were performed using Mascot software (version 2.3.01; Matrix Science, Inc., Boston, MA, USA). The raw MS/MS data of the iTRAQ-labeled peptides was first converted into an msg file and then searched against the NCBI human reference sequence database (www.ncbi.nlm.nih.gov, downloaded on the 5th December 2011 with 216,686 sequences). The database-searching parameters were as follows: Carbamidomethyl (C) for fixed modifications, oxidation (M), Gln->Pyro-Glu (N-term Q), iTRAQ8plex (K), iTRAQ8plex (Y), and iTRAQ8plex (N-term) for variable modification, 1 for maximum missed cleavages, and $15 \mathrm{ppm}$ and $20 \mathrm{mmu}$ for peptide and MS/MS tolerance, respectively. The iTRAQ8plex modification rate of the spectrum was used for monitoring the labeling efficiency rate. For quantitative analysis, the protein ratio type was set at the median, the minimum unique peptides at 2 and the normalization method was the median ratio. $\mathrm{P}<0.05$ was considered to indicate a statistically significant difference. The differential threshold was set at 1.5. Student's t-tests were performed using R software (version 3.2.4; https://www.r-project.org). 
Table I. Differentially expressed proteins identified in PCA.

\begin{tabular}{|c|c|c|c|c|}
\hline Accession no. & Gene abbreviation & Gene name & Expression ratio $^{\mathrm{a}}$ & P-value \\
\hline 4557581 & FABP5 & Fatty acid-binding protein, epidermal & 2.990 & $6.05 \times 10^{-10}$ \\
\hline 1195531 & KRT16 & Type I keratin 16 & 2.757 & $1.61 \times 10^{-6}$ \\
\hline 189053201 & S100A9 & S100 calcium-binding protein A9 & 2.602 & $9.96 \times 10^{-26}$ \\
\hline 223278387 & CALML5 & $\begin{array}{l}\text { Calmodulin-like protein } 5 \\
\text { (calmodulin-like skin protein) }\end{array}$ & 2.598 & $3.50 \times 10^{-4}$ \\
\hline 21614544 & S100A8 & S100 calcium-binding protein A8 & 2.557 & $1.32 \times 10^{-6}$ \\
\hline 12053626 & S100A7 & Psoriasin (S100 calcium-binding protein A7) & 2.315 & $2.11 \times 10^{-24}$ \\
\hline 194387918 & KRT6A & Keratin, type II cytoskeletal 6A & 2.088 & $4.70 \times 10^{-7}$ \\
\hline 553348 & $\mathrm{TNC}$ & Hexabrachion & 1.992 & $1.51 \times 10^{-5}$ \\
\hline 457464 & DSC1 & Dsc1a precursor (desmocollin 1) & 1.983 & $4.18 \times 10^{-3}$ \\
\hline 124056490 & COL3A1 & Collagen $\alpha 1$ (III) chain & 1.845 & $3.57 \times 10^{-61}$ \\
\hline 6912286 & CASP14 & Caspase-14 precursor & 1.834 & $2.08 \times 10^{-12}$ \\
\hline 49660012 & TPM1 & Sarcomeric tropomyosin $\kappa$ & 1.821 & $2.05 \times 10^{-3}$ \\
\hline 1942977 & MIF & Chain a, macrophage migration inhibitory factor & 1.816 & $4.84 \times 10^{-7}$ \\
\hline 4503113 & CST6 & Cystatin-M precursor & 1.809 & $1.39 \times 10^{-2}$ \\
\hline 110349772 & COL1A1 & Collagen $\alpha 1$ (I) chain preproprotein & 1.744 & $0.00 \times 10^{0}$ \\
\hline 115527066 & COL6A2 & Collagen $\alpha 2$ (VI) chain isoform $2 \mathrm{C} 2 \mathrm{a}$ precursor & 1.734 & $1.52 \times 10^{-3}$ \\
\hline 178849 & APOE & Apolipoprotein E & 1.720 & $2.47 \times 10^{-7}$ \\
\hline 119585301 & COL7A1 & $\begin{array}{l}\text { Collagen, type VII, } \alpha 1 \text { (epidermolysis bullosa, } \\
\text { dystrophic, dominant and recessive), isoform CRA_e }\end{array}$ & 1.703 & $1.26 \times 10^{-3}$ \\
\hline 4505047 & LUM & Lumican precursor & 1.700 & $7.83 \times 10^{-168}$ \\
\hline 453157 & FMOD & Fibromodulin & 1.689 & $1.68 \times 10^{-2}$ \\
\hline 48255905 & TAGLN & Transgelin (SM22) & 1.683 & $1.10 \times 10^{-18}$ \\
\hline 183583553 & COL4A5 & Collagen $\alpha 5$ (VI) chain & 1.681 & $2.29 \times 10^{-2}$ \\
\hline 21708099 & DPT & Dermatopontin & 1.647 & $6.17 \times 10^{-12}$ \\
\hline 32451581 & COL1A2 & Collagen, type I, $\alpha 2$ & 1.640 & $1.03 \times 10^{-138}$ \\
\hline 4885165 & CSTA & Cystatin-A & 1.567 & $1.61 \times 10^{-2}$ \\
\hline 166343769 & gil166343769 & Periostin isoform thy 6 & 1.557 & $5.03 \times 10^{-20}$ \\
\hline 49456765 & $\mathrm{SFN}$ & Stratifin & 1.519 & $1.12 \times 10^{-20}$ \\
\hline 113414871 & KRT77 & Keratin 77 (type II) & 0.664 & $2.24 \times 10^{-2}$ \\
\hline 126035028 & HIST1H1E & Liver histone $\mathrm{H} 1 \mathrm{e}$ & 0.646 & $2.35 \times 10^{-4}$ \\
\hline 119573007 & APOA2 & Apolipoprotein A-II, isoform CRA_d & 0.644 & $4.99 \times 10^{-13}$ \\
\hline 221045918 & TUBB4B & Tubulin $\beta-4 \mathrm{~B}$ chain & 0.643 & $1.77 \times 10^{-5}$ \\
\hline 167614485 & ACAA2 & 3-ketoacyl-coa thiolase, mitochondrial & 0.642 & $5.18 \times 10^{-5}$ \\
\hline 9857759 & gil9857759 & Recombinant IgG4 heavy chain & 0.633 & $1.53 \times 10^{-50}$ \\
\hline 193785892 & ATP2A1 & $\begin{array}{l}\text { Sarcoplasmic/endoplasmic reticulum } \\
\text { calcium ATPase } 1\end{array}$ & 0.631 & $1.55 \times 10^{-3}$ \\
\hline 4502011 & AK1 & Adenylate kinase isoenzyme 1 & 0.620 & $2.98 \times 10^{-3}$ \\
\hline 221041416 & ACSL1 & Long-chain-fatty-acid-coA ligase 1 & 0.619 & $2.69 \times 10^{-3}$ \\
\hline 297304885 & FHL1 & $\begin{array}{l}\text { PREDICTED: Four and a half LIM domains } \\
\text { protein } 1\end{array}$ & 0.613 & $3.48 \times 10^{-3}$ \\
\hline 194387956 & ADH1B & Alcohol dehydrogenase 1B & 0.593 & $6.27 \times 10^{-17}$ \\
\hline 61743954 & AHNAK & $\begin{array}{l}\text { Neuroblast differentiation-associated } \\
\text { protein AHNAK isoform } 1\end{array}$ & 0.587 & $5.05 \times 10^{-126}$ \\
\hline 109731497 & MYH1 & Myosin, heavy chain 1 , skeletal muscle, adult & 0.573 & $3.85 \times 10^{-5}$ \\
\hline 4506335 & PVALB & Parvalbumin $\alpha$ & 0.518 & $3.43 \times 10^{-2}$ \\
\hline 194374151 & ENO3 & $\beta$-enolase; 2 -phospho-D-glycerate hydro-lyase & 0.504 & $9.95 \times 10^{-4}$ \\
\hline 34071 & KRT15 & Keratin 15 (type I) & 0.501 & $2.98 \times 10^{-16}$ \\
\hline 153791586 & MYH2 & Myosin-2 & 0.492 & $1.16 \times 10^{-28}$ \\
\hline 109111058 & TPM2 & PREDICTED: $\beta$-tropomyosin isoform & 0.445 & $1.22 \times 10^{-2}$ \\
\hline 825683 & TMSB4X & Thymosin $\beta 4$ & 0.427 & $1.05 \times 10^{-2}$ \\
\hline 4507617 & TNNC2 & Troponin C, skeletal muscle & 0.421 & $3.93 \times 10^{-7}$ \\
\hline
\end{tabular}


Table I. Continued.

\begin{tabular}{|c|c|c|c|c|}
\hline Accession no. & Gene abbreviation & Gene name & Expression ratio $^{\mathrm{a}}$ & P-value \\
\hline 28372499 & MYL2 & $\begin{array}{l}\text { Myosin regulatory light chain } 2 \text {, skeletal } \\
\text { muscle isoform }\end{array}$ & 0.412 & $5.07 \times 10^{-16}$ \\
\hline 168985192 & MB & Myoglobin & 0.356 & $8.45 \times 10^{-6}$ \\
\hline 189053833 & CKM & Creatine kinase, muscle (CKM) & 0.354 & $1.04 \times 10^{-11}$ \\
\hline 17986273 & MYL1 & $\begin{array}{l}\text { Myosin light chain } 1 / 3 \text {, skeletal Muscle } \\
\text { isoform isoform 1f }\end{array}$ & 0.304 & $2.95 \times 10^{-15}$ \\
\hline
\end{tabular}

aProtein ratio of the PCA group vs. the control group. PCA, primary cutaneous amyloidosis.

IHC analysis. Paraffin-embedded skin tissues were sliced into $3-\mu$ m-thick sections and mounted on glass slides. IHC was performed according to the protocols provided by the manufacturers of the primary antibodies (Abcam, Cambridge, UK) and PowerVision ${ }^{\mathrm{TM}}$ Two-Step Histostaining reagent (OriGene Technologies, Inc., Beijing, China). After de-waxing (placed in a $65^{\circ} \mathrm{C}$ incubator for $30 \mathrm{~min}$, twice in dimethylbenzene at room temperature for $30 \mathrm{~min}$ ), rehydration $(5 \mathrm{~min}$ for each incubation at room temperature with descending ethanol from $100,95,85$, to a final $75 \%$ ), antigen retrieval and $5 \%$ bovine serum albumin (Sigma-Aldrich; Merck KGaA, Darmstadt, Germany) blocking at room temperature for $1 \mathrm{~h}$, the slides were incubated with primary antibodies (1:5,000 anti-S100A7 ab83543, 1:20,000 anti-S100A8 ab92331, and 1:20,000 anti-CALML5 ab154631) at $4^{\circ} \mathrm{C}$ overnight, followed by incubation with PowerVision ${ }^{\mathrm{TM}}$ Two-Step Histostaining reagent at room temperature for $20 \mathrm{~min}$. The slides were then stained with 3,3'-diaminobenzidine for $5 \mathrm{~min}$ and hematoxylin for 2 min at room temperature. An Olympus BX-51 microscope (Olympus Corporation, Tokyo, Japan) equipped with a digital camera system was used to capture images of the slides. IHC staining on epidermal cells was quantified using ImageJ v1.50 software (National Institutes of Health, Bethesda, MD, USA). Threshold was determined for each target protein at the point where true negatives were about to show color. The percentage of the area that met the threshold was multiplied by the mean intensity of the area to generate an integrated score. Wilcoxon signed-rank tests were performed to compare IHC results between the two groups, using $\mathrm{R}$ software. $\mathrm{P}<0.05$ was considered to indicate a statistically significant difference.

Bioinformatics analysis. Gene ontology (GO) term and Kyoto Encyclopedia of Genes and Genomes (KEGG) pathway enrichment analyses were performed using a gene list enrichment analysis tool, Enrichr (http://amp.pharm.mssm. edu/Enrichr) (18). The output of Enrichr provides adjusted $\mathrm{P}$ values computed using the Fisher exact test, $\mathrm{Z}$ score computed by assessing the deviation from the expected rank, and combined score from multiplying $\log$ (adjusted $\mathrm{P}$-value) and $\mathrm{Z}$ score. Adjusted $\mathrm{P}<0.001$ was considered to indicate a statistically significant difference. Protein-protein interaction (PPI) analysis was performed using a gene interaction analysis tool, genes2FANs (http://actin.pharm.mssm.edu/genes2FANs) (19). A maximum path length of 2 and significance cutoff of 2.5 were used.

\section{Results}

Identification of differentially expressed proteins in PCA skin tissue. To identify proteins that are differentially expressed in PCA skin tissue, comparative proteomic analysis was performed on pooled diseased and normal skin tissues. Proteins extracted from skin tissues were trypsin-digested and labeled with iTRAQ reagents. The labeled samples from diseased and normal skin tissues were then combined prior to LC-MS/MS analysis. A total of 318,710 spectra were captured and 1,032 proteins were identified, with a false discovery rate of $<1 \%$. Among them, 533 proteins had at least two unique peptides and were subject to quantitative comparison. Using a strict 1.5 -fold change and $\mathrm{P}<0.05$ threshold, 51 proteins were identified to be differentially expressed between diseased and normal skin tissue (Table I). Among these, 27 were upregulated and 24 were downregulated in PCA lesions compared with normal skin tissue.

Analysis of differentially expressed proteins in PCA lesions. Subcellular distribution analysis of the differentially expressed proteins identified in PCA (Fig. 1A) revealed that the upregulated proteins were markedly enriched in the plasma membrane (48\%), among which one known amyloid protein, ApoE, was identified (Table I). Only one plasma membrane protein was identified out of the downregulated proteins $(4 \%)$, while the majority of downregulated proteins were cytoplasmic $(72 \%)$.

GO term enrichment analysis suggested that upregulated proteins in PCA (Fig. 1B) were significantly enriched in seven biological processes (adjusted $\mathrm{P}<0.001$ ). The processes with the highest combined score were tissue development (GO:0009888) and epidermis development (GO:0008544), which both included S100 calcium-binding protein A7 (S100A7), collagen type III $\alpha 1$ chain (COL3A1), COL7A1, calmodulin-like protein 5 (CALML5), caspase-14 precursor and fatty acid-binding protein, epidermal (FABP5). The processes with the next highest scores were anatomical structure development (GO:0048856), collagen fibril organization (GO:0030199), response to reactive oxygen species (GO:0000302), response to wounding (GO:0009611) and ECM organization (GO:0030198). The only significantly enriched molecular function of the upregulated proteins in PCA was calcium ion binding (GO:0005509; adjusted $\mathrm{P}=0.004$; data not shown). For downregulated proteins in PCA, the most 
A

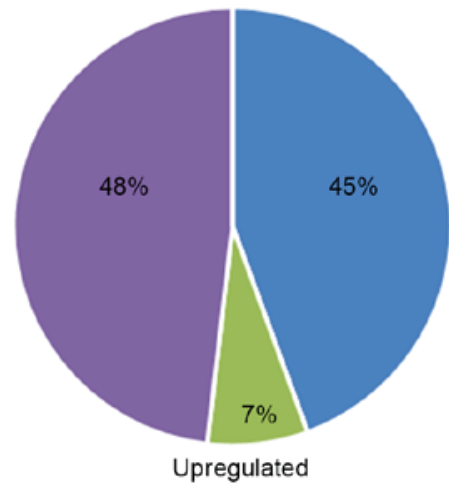

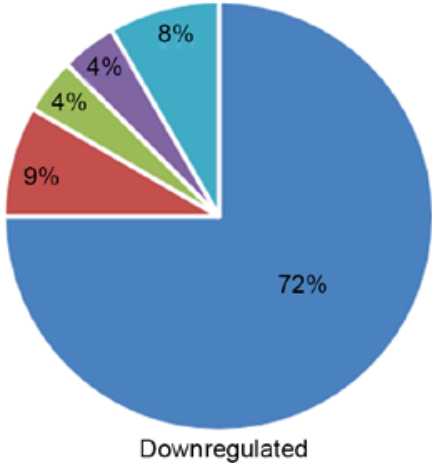

Downregulated
Cytoplasm

nucleus

Extracellular space

- Plasma membrane

Secreted

B

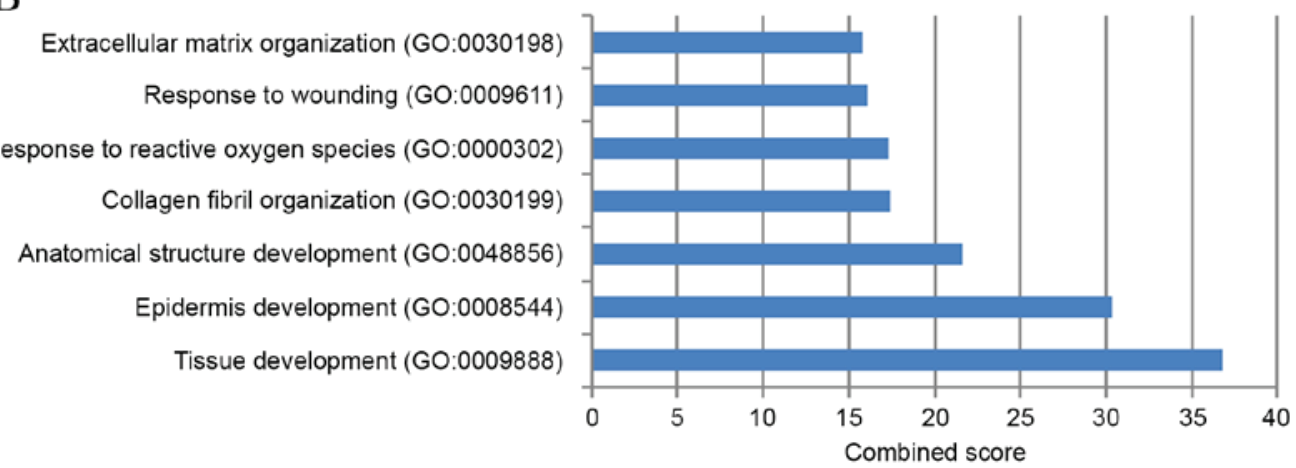

Figure 1. Subcellular distribution and functional analysis of differentially expressed proteins in PCA. (A) Subcellular distribution of differentially expressed proteins in PCA lesions. Pie charts indicate the percentage of various subcellular locations of upregulated (left) and downregulated (right) proteins. (B) Biological processes that the upregulated proteins in PCA were significantly enriched in (adjusted $\mathrm{P}<0.001$ ). PCA, primary cutaneous amyloidosis; GO, gene ontology.

enriched cellular process was regulation of muscle contraction (GO:0006937; adjusted $\mathrm{P}=0.003$ ) and structural constituent of muscle (GO:0008307; adjusted $\mathrm{P}<0.001$ ) (data not shown).

KEGG pathway enrichment analysis revealed that the upregulated proteins in PCA were enriched in three pathways as follows: Cell communication, ECM receptor interaction and focal adhesion (adjusted $\mathrm{P}<0.001$; data not shown). The proteins involved in these three signaling pathways include COL3A1, COL6A2, COL1A2, COL1A1 and hexabrachion (TNC). No significantly enriched signaling pathways were identified for the downregulated proteins.

PPI analysis using a maximum path length of 2 and significance cutoff of 2.5 revealed two clusters of interaction among upregulated proteins (Fig. 2A). One of these included COL3A1, COL7A1, COL1A1, COL1A2, COL4A5 and $\mathrm{TNC}$, the majority of which are involved in focal adhesion according to enrichment analysis. Within this cluster, COL1A1, COL1A2 and TNC directly interact with integrin $\alpha 2$ (ITGA2), the receptor in focal adhesion. The second cluster included S100A9, S100A8, S100A7, FABP5, macrophage migration inhibitory factor and CALML5, among which S100A9 and S100A8, and S100A7 and FABP5 interact directly. The majority of the proteins in this second cluster function in calcium ion binding according to enrichment analysis. For downregulated proteins, there were also two clusters of interaction (Fig. 2B). One cluster contained 6 downregulated proteins that formed a network through 4 intermediate proteins that interacted with one another. Troponin $\mathrm{C}$ interacts with troponin I fast skeletal muscle isoform $2, \beta$-enolase and creatinine kinase muscle (CKM) interact with tripartite motif-containing protein 63; CKM, four and a half LIM domains protein 1 (FHL1) and adenylate kinase isoenzyme 1 all interact with FHL2; and $\beta$-tropomyosin interacts with JUN. In the second cluster, a downregulated protein tubulin $\beta-4 \mathrm{~B}$ chain interacted with another 2 downregulated proteins, myosin light chain $1 / 3$ and alcohol dehydrogenase $1 \mathrm{~B}$, through the intermediate proteins interleukin 7 receptor and 14-3-3 protein $\varepsilon$, respectively.

Distribution of upregulated calcium ion binding proteins in PCA. Calcium ion binding was identified as the most enriched molecular function associated with the upregulated proteins in PCA, and the calcium binding proteins CALML5, S100A7/FABP5 and S100A8/A9 were among the most upregulated proteins in the PCA samples, with fold changes $>2$. To validate these results and to observe the distribution of these proteins in epidermal layers, IHC analysis for CALML5, S100A7 and S100A8 was performed on skin tissues from 29 LA and 17 control samples. Fig. 3A shows representative IHC staining images and Fig. 3B presents quantification of the IHC results. The staining for all three proteins in PCA skin tissues was significantly stronger compared with normal skin tissues $(\mathrm{P}<0.05)$, which was consistent with the proteomic results. S100A7 and S100A8 were expressed in the cornified and granular layers in PCA lesions, while they were generally absent in normal skin. CALML5 was expressed primarily in the outermost cornified layer in normal skin, while it was expressed in the granular layers in PCA. 
A
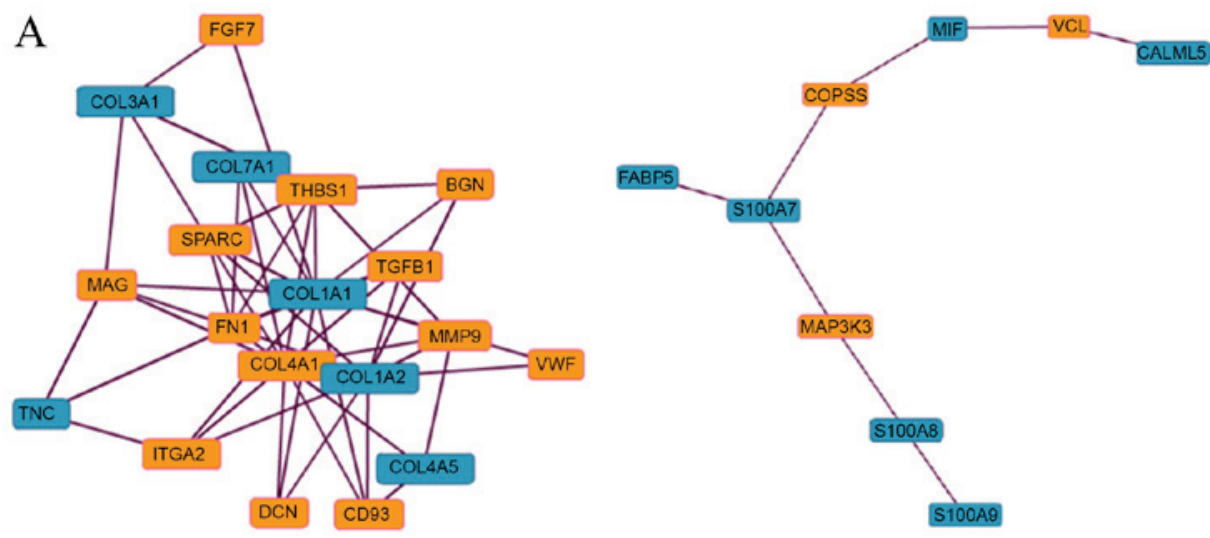

B

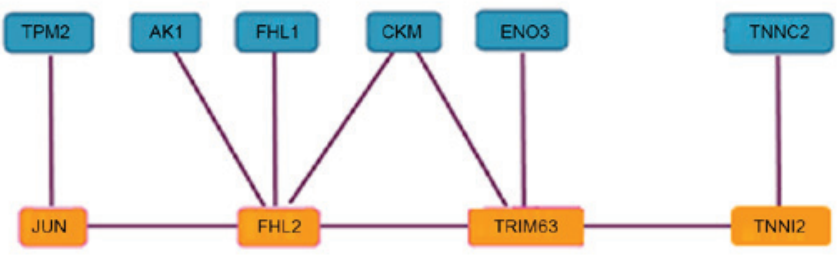

Figure 2. PPI analysis of differentially expressed proteins in PCA. Proteins that were (A) upregulated and (B) downregulated proteins in PCA were subjected to PPI analysis using a maximum path length of 2 and significance cutoff of 2.5 . Blue and orange boxes indicate proteins that were and were not identified as differentially expressed proteins, respectively. PPI, protein-protein interaction; PCA, primary cutaneous amyloidosis.

\section{Discussion}

In the present study, comparative proteomics technology was used to identify 51 differentially expressed proteins in PCA lesions. Three of the upregulated proteins identified were also verified by IHC analysis. In PCA lesions, the majority of upregulated proteins were located extracellularly. It was demonstrated that the upregulated proteins were associated with the biological processes of epidermal development, collagen fiber organization and response to wounding, and the molecular function of calcium ion binding. The upregulated proteins were also significantly enriched in the signaling pathways of cell communication, ECM receptor interaction and focal adhesion.

Amyloid deposition in the ECM is the key feature of PCA as well as all other amyloidosis. Among all reported PCA amyloid components, only ApoE was demonstrated to be upregulated in PCA lesions in the present study. ApoE is an apolipoprotein that transports cholesterol and triglycerides in the blood (20). The function of ApoE in Alzheimer's disease, an amyloidosis of the brain, is to affect the fate of amyloid $\beta$ $(A \beta)$, which is deposited into the extracellular space after it undergoes a configuration change (21). However, the deposition of $\mathrm{A} \beta$ has not previously been reported in PCA. Whether ApoE serves a similar function to other amyloid proteins in PCA requires further study.

CKs are known to be the major amyloid proteins in PCA. The following four CKs have been reported to be present in amyloid deposits in descending order of abundance: CK5,
CK1, CK14 and CK10 (22). In the present study, these four CKs were identified in PCA lesions; however, their abundance was not significantly different between the PCA and control samples (fold change, 0.82-1.22), which could be due to the fact that whole tissue lysates rather than isolated amyloid deposits were used.

In the present study, half of the upregulated extracellular proteins (6/12) in PCA lesions were collagens, which were identified to be enriched in the signaling pathways of cell communication, ECM receptor interaction and focal adhesion. Focal adhesions mediate a dynamic connection between cells and the ECM, functioning as a mechanical connection and biological signaling center. Integrins serve as mediators in focal adhesion, connecting extracellular collagens to proteins inside the cells. PPI analysis revealed that two upregulated collagens in PCA, COL1A1 and COL1A2, could directly bind to ITGA2. In addition to the six collagens, five additional upregulated extracellular proteins were associated with collagen function. TNC, a large ECM glycoprotein, is a component in focal adhesion and can bind to the ITGA2 (23). Lumican precursor (LUM), a dermatan sulfate proteoglycan, regulates the assembly of collagen microfibers (24). Fibromodulin (FMOD), a small leucine-rich glycoprotein, regulates the generation of collagen microfibers (25). Dermatopontin (DPT), a widely distributed small ECM protein, is primarily located on the surface of collagen fibers in skin where it exerts cell adhesion activity (26). Periostin, also called osteoblast-specific factor 2, regulates collagen fibrillogenesis (27). Periostin can also facilitate 
A
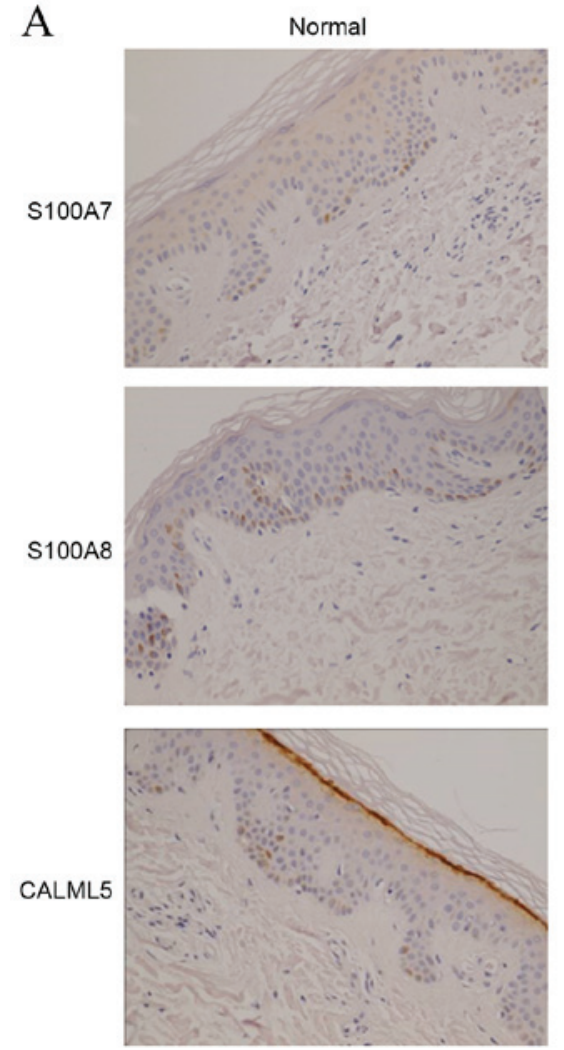

PCA
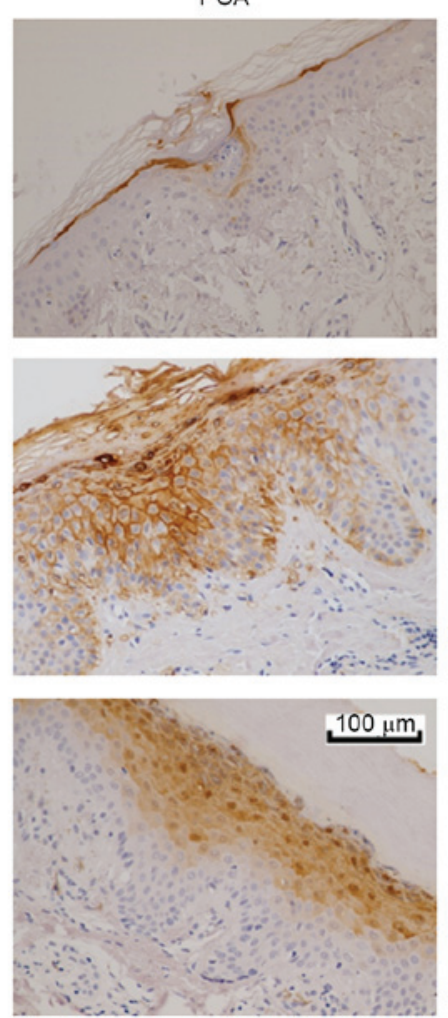

B
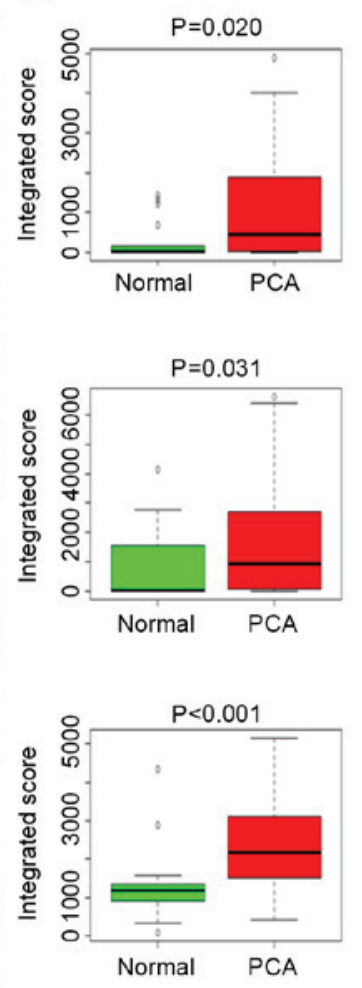

Figure 3. IHC validation of selected differentially expressed proteins in PCA. (A) Representative images of IHC analysis. Skin samples from normal controls $(\mathrm{n}=17)$ and patients with PCA $(\mathrm{n}=29)$ were subjected to S100A7, S100A8 or CALML5 staining. (B) Box and whisker plots of integrated scores from the IHC staining of epidermal cells in the normal and PCA groups. S100A7/8, S100 calcium-binding protein A7/8; CALML5, calmodulin-like protein 5; IHC, immunohistochemistry; PCA, primary cutaneous amyloidosis.

integrin-dependent cell adhesion and mobility through the binding of its C-terminal domain to ECM proteins, including collagens and fibronectin. Different isoforms of periostin have distinct effects on the formation of ECM fibers (28). A total of $41 \%$ (11/27) of the upregulated proteins identified in PCA lesions in the present study were collagens or functional components in collagen fiber organization, suggesting that focal adhesion mediated by collagens serves a key role in PCA.

Calcium ion binding was identified as the top enriched molecular function associated with upregulated proteins in PCA in the present study, and the calcium binding proteins CALML5, S100A7/FABP5 and S100A8/A9 were among the top upregulated proteins, with a fold change $>2$. This may indicate the presence of a high $\mathrm{Ca}^{2+}$ concentration in PCA lesions. Calcium ions serve key functions in numerous cellular processes. Thus, as $\mathrm{Ca}^{2+}$ sensors and transductors, calcium-binding proteins are involved in numerous biological processes (29). For example, S100A7, a member of the S100 protein family, regulates essential cellular activities, including proliferation, differentiation, apoptosis, signal transduction and lipid metabolism. S100A7 is also involved in cell adhesion and migration as a substrate of transglutaminase, and is a precursor of cornified envelope formation (30). FABP5, the binding partner of S100A7, forms a complex with S100A7 in the cytoplasm in the absence of $\mathrm{Ca}^{2+}(31)$. Upon treatment with $\mathrm{Ca}^{2+}$, the complex moves to the outer region of the cell where it colocalizes with $\alpha$-actinin and paxillin in a focal adhesion-like structure (32). S100A8 forms a heterodimer with S100A9 in a $\mathrm{Ca}^{2+}$-dependent manner, which is involved in inflammation, regulating cell growth and differentiation, inhibiting cell proliferation and inducing apoptosis (33). CALML5, also known as calmodulin-like skin protein, serves a function in keratinocyte differentiation (34). CALML5 is primarily located in the cornified and granular layers in the epidermis, and CALML5 positive cells are typically undergoing differentiation rather than proliferation (35). Using IHC in the present study, it was identified that CALML5 positive cells expanded from the outer cornified layer in normal skin to the granular layers in PLA lesions, which indicates an elevation of differentiation in PCA. An increase in differentiation in the epidermis of PCA lesions was also indicated in previous studies by the elevation of several keratinocyte differentiation markers, including desmocollin-1 (36), cystatin-M precursor (37) and Caspase-14 (38). Another indicator of increased differentiation is the downregulation of keratin 15 (KRT15), as observed in the present study, because its expression is normally limited to proliferating keratinocytes in the basal layer and is reduced when cells start to differentiate and move outwards (39).

A key pathogenic factor in PCA is prolonged and repeated friction, which may cause small wounds in the skin and trigger the wound healing process. $\mathrm{GO}$ analysis performed in the present study demonstrated that the upregulated proteins in PCA were significantly enriched in the process of response to wounding, suggesting that the wound healing process was triggered. The enriched proteins included S100A8, S100A9, TNC, COL3A1 and sarcomeric tropomyosin $\kappa$. Several 
additional proteins that have previously been reported to be involved in wound healing were identified to be upregulated in PCA in the present study, including TNC (40), LUM (24), FMOD (25) and DPT (26). In addition, the downregulation of KRT15 in keratinocytes has previously been reported during wound healing (41) and was demonstrated to be downregulated in PCA lesions in the present study. These results suggest a wound healing process occurs in PCA lesions. However, whether this is a causative association or not remains unclear.

In conclusion, the current study identified numerous proteins that are differentially expressed in PCA. Analysis of the upregulated proteins suggests a possible increase in focal adhesion, differentiation and wound healing activities, and a possible increase in $\mathrm{Ca}^{2+}$ concentration, which is consistent with the symptom of skin hyperplasia and the key pathogenic factor of prolonged friction. The present study expands the knowledge of the molecular basis of PCA and provides novel insights into the pathology of the disease.

\section{Acknowledgements}

The present study was supported by Shandong Province Science and Technology Development Projects (grant no. 2015GSF118060), and the Science Foundation of Qilu Hospital of Shandong University (grant no. 2015QLMS16).

\section{References}

1. Pinney JH and Hawkins PN: Amyloidosis. Ann Clin Biochem 49: 229-241, 2012.

2. Zhao B: The Chinese clinical dermatology. Phoenix Science press: Nanjing, pp1395-1401, 2010 (In Chinese).

3. Fang S, Shen X, Chen AJ, Li S and Shan K: Health-related quality of life in patients with primary cutaneous amyloidosis. PLoS One 10: e0120623, 2015.

4. Krishna A, Nath B, Dhir GG, Kumari R, Budhiraja V and Singh K: Study on epidemiology of cutaneous amyloidosis in northern India and effectiveness of dimethylsulphoxide in cutaneous amyloidosis. Indian Dermatol Online J 3: 182-186, 2012.

5. Tanaka A, Arita K, Lai-Cheong JE, Palisson F, Hide M and McGrath JA: New insight into mechanisms of pruritus from molecular studies on familial primary localized cutaneous amyloidosis. Br J Dermatol 161: 1217-1224, 2009.

6. Brambilla F, Lavatelli F, Merlini G and Mauri P: Clinical proteomics for diagnosis and typing of systemic amyloidosis. Proteomics Clin Appl 7: 136-143, 2013.

7. Apaydin R, Gürbüz Y, Bayramgürler D, Müezzinoglu B and Bilen N: Cytokeratin expression in lichen amyloidosis and macular amyloidosis. J Eur Acad Dermatol Venereol 18: 305-309, 2004

8. Furumoto H, Shimizu T, Asagami C, Muto M, Takahashi M, Hoshii Y, Ishihara T and Nakamura K: Apolipoprotein E is present in primary localized cutaneous amyloidosis. J Invest Dermatol 111: 417-421, 1998.

9. Furumoto H, Hashimoto Y, Muto M, Shimizu T and Nakamura K: Apolipoprotein E4 is associated with primary localized cutaneous amyloidosis. J Invest Dermatol 119: 532-533, 2002.

10. Miura Y, Harumiya S, Ono K, Fujimoto E, Akiyama M, Fujii N, Kawano $\mathrm{H}$, Wachi $\mathrm{H}$ and Tajima $\mathrm{S}$ : Galectin-7 and actin are components of amyloid deposit of localized cutaneous amyloidosis. Exp Dermatol 22: 36-40, 2013.

11. Saeedi M, Ebrahim-Habibi A, Haghighi A, Zarrabi F, Amoli MM and Robati RM: A novel missense mutation in oncostatin M receptor beta causing primary localized cutaneous amyloidosis. Biomed Res Int 2014: 653724, 2014

12. Lin MW, Lee DD, Liu TT, Lin YF, Chen SY, Huang CC, Weng HY, Liu YF, Tanaka A, Arita K, et al: Novel IL31RA gene mutation and ancestral OSMR mutant allele in familial primary cutaneous amyloidosis. Eur J Hum Genet 18: 26-32, 2010 .
13. Tanaka A, Lai-Cheong JE, van den Akker PC, Nagy $\mathrm{N}$, Millington G, Diercks GF, van Voorst Vader PC, Clements SE, Almaani N, Techanukul T, et al: The molecular skin pathology of familial primary localized cutaneous amyloidosis. Exp Dermatol 19: 416-423, 2010.

14. Lu H, Yang Y, Allister EM, Wijesekara N and Wheeler MB: The identification of potential factors associated with the development of type 2 diabetes: A quantitative proteomics approach. Mol Cell Proteomics 7: 1434-1451, 2008.

15. Masui O, White NM, DeSouza LV, Krakovska O, Matta A, Metias S, Khalil B, Romaschin AD, Honey RJ, Stewart R, et al: Quantitative proteomic analysis in metastatic renal cell carcinoma reveals a unique set of proteins with potential prognostic significance. Mol Cell Proteomics 12: 132-144, 2013.

16. Bradford MM: A rapid and sensitive method for the quantitation of microgram quantities of protein utilizing the principle of protein-dye binding. Anal Biochem 72: 248-254, 1976.

17. Chen X, Zhu Y, Wang Z, Zhu H, Pan Q, Su S, Dong Y, Li L, Zhang $\mathrm{H}, \mathrm{Wu} \mathrm{L}$, et al: mTORC1 alters the expression of glycolytic genes by regulating KPNA2 abundances. J Proteomics 136: 13-24, 2016.

18. Chen EY, Tan CM, Kou Y, Duan Q, Wang Z, Meirelles GV, Clark NR and Ma'ayan A: Enrichr: Interactive and collaborative HTML5 gene list enrichment analysis tool. BMC Bioinformatics 14: 128, 2013.

19. Dannenfelser R, Clark NR and Ma'ayan A: Genes2FANs: Connecting genes through functional association networks. BMC Bioinformatics 13: 156, 2012.

20. Mahley RW: Apolipoprotein E: Cholesterol transport protein with expanding role in cell biology. Science 240: 622-630, 1988.

21. Liu CC, Liu CC, Kanekiyo T, Xu H and Bu G: Apolipoprotein E and Alzheimer disease: Risk, mechanisms and therapy. Nat Rev Neurol 9: 106-118, 2013.

22. Chang YT, Liu HN, Wang WJ, Lee DD and Tsai SF: A study of cytokeratin profiles in localized cutaneous amyloidsis. Arch Dermatol Res 296: 83-88, 2004.

23. Midwood KS and Orend G: The role of tenascin-C in tissue injury and tumorigenesis. J Cell Commun Signal 3: 287-310, 2009.

24. Liu XJ, Kong FZ, Wang YH, Zheng JH, Wan WD, Deng CL, Mao GY, Li J, Yang XM, Zhang YL, et al: Lumican accelerates wound healing by enhancing $\alpha 2 \beta 1$ integrin-mediated fibroblast contractility. PLoS One 8: e67124, 2013.

25. Zheng Z, Nguyen C, Zhang X, Khorasani H, Wang JZ, Zara JN, Chu F, Yin W, Pang S, Le A, et al: Delayed wound closure in fibromodulin-deficient mice is associated with increased TGF- $\beta 3$ signaling. J Invest Dermatol 131: 769-778, 2011.

26. Okamoto O and Fujiwara S: Dermatopontin, a novel player in the biology of the extracellular matrix. Connect Tissue Res 47: 177-189, 2006.

27. Norris RA, Damon B, Mironov V, Kasyanov V, Ramamurthi A, Moreno-Rodriguez R, Trusk T, Potts JD, Goodwin RL, Davis $\mathrm{J}$, et al: Periostin regulates collagen fibrillogenesis and the biomechanical properties of connective tissues. J Cell Biochem 101: 695-711, 2007.

28. Morra L and Moch H: MPeriostin expression and epithelial-mesenchymal transition in cancer: A review and an update. Virchows Arch 459: 465-475, 2011.

29. Méhul B, Bernard D, Brouard M, Delattre C and Schmidt R: Influence of calcium on the proteolytic degradation of the calmodulin-like skin protein (calmodulin-like protein 5) in psoriatic epidermis. Exp Dermatol 15: 469-477, 2006.

30. Ruse M, Lambert A, Robinson N, Ryan D, Shon KJ and Eckert RL: S100A7, S100A10, and S100A11 are transglutaminase substrates. Biochemistry 40: 3167-3173, 2001.

31. Hagens G, Masouyé I, Augsburger E, Hotz R, Saurat JH and Siegenthaler G: Calcium-binding protein S100A7 and epidermal-type fatty acid-binding protein are associated in the cytosol of human keratinocytes. Biochem J 339: 419-427, 1999.

32. Méhul B, Bernard D and Schmidt R: Calmodulin-like skin protein: A new marker of keratinocyte differentiation. J Invent Dermatol 116: 905-909, 2001.

33. Kerkhoff C, Klempt M and Sorg C: Novel insights into structure and function of MRP8 (S100A8) and MRP14 (S100A9). Biochim Biophys Acta 1448: 200-211, 1998.

34. Méhul B, Bernard D, Simonetti L, Bernard MA and Schmidt R: Identification and cloning of a new calmodulin-like protein from human epidermis. J Biol Chem 275: 12841-12847, 2000.

35. Watson PH, Leygue ER and Murphy LC: Psoriasin (S100A7). Int J Biochem Cell Biol 30: 567-571, 1998. 
36. Henkler F, Strom M, Mathers K, Cordingley H, Sullivan K and King I: Trangenic misexpression of the differentiation-specific desmocollin isoform 1 in basal keratinocytes. J Invest Dermatol 116: 144-149, 2001

37. Zeeuwen PL, van Vlijmen-Willems IM, Cheng $T$, Rodijk-Olthuis D, Hitomi K, Hara-Nishimura I, John S, Smyth N, Reinheckel T, Hendriks WJ and Schalkwijk J: The cystatin $\mathrm{M} / \mathrm{E}$ - cathepsin L balance is essential for tissue homeostasis in epidermis, hair follicles, and cornea. FASEB J 24: 3744-3755, 2010.

38. Brocklehurst K and Philpott MP: Cysteine proteases: Mode of action and role in epidermal differentiation. Cell Tissue Res 351: 237-244, 2013
39. Bose A, Teh MT, Mackenzie IC and Waseem A: Keratin k15 as a biomarker of epidermal stem cells. Int J Mol Sci 14: 19385-19398, 2013.

40. Ogawa K, Ito M, Takeuchi K, Nakada A, Heishi M, Suto H, Mitsuishi K, Sugita Y, Ogawa $H$ and Ra C: Tenascin-C is upregulated in the skin lesions of patients with atopic dermatitis. J Dermatol Sci 40: 35-41, 2005.

41. Werner S and Munz B: Suppression of keratin 15 expression by transforming growth factor beta in vitro and by cutaneous injury in vivo. Exp Cell Res 254: 80-90, 2000. 